\title{
Parahippocampal deactivation and hyperactivation of central executive, saliency and social cognition networks in autism spectrum disorder
}

\author{
Susana Mouga $a^{1,2,3}$, Isabel Catarina Duarte ${ }^{1,2}$, Cátia Café ${ }^{3}$, Daniela Sousa ${ }^{1,2,3}$, Frederico Duque $^{3,4}$, \\ Guiomar Oliveira ${ }^{1,3,4+}$ and Miguel Castelo-Branco ${ }^{1,2,4^{*}+}$ (i)
}

\begin{abstract}
Background: The concomitant role of the Central Executive, the Saliency and the Social Cognition networks in autism spectrum disorder (ASD) in demanding ecological tasks remains unanswered. We addressed this question using a novel task-based fMRI virtual-reality task mimicking a challenging daily-life chore that may present some difficulties to individuals with ASD: the EcoSupermarketX.

Methods: Participants included 29 adolescents: 15 with ASD and 15 with typical neurodevelopment (TD). They performed the EcoSupermarketX (a shopping simulation with three goal-oriented sub-tasks including "no cue","nonsocial" or "social" cues), during neuroimaging and eye-tracking.

Results: ASD differed from TD only in total time and distance to complete the "social cue" sub-task with matched eye-tracking measures. Neuroimaging revealed simultaneous hyperactivation across social, executive, and saliency circuits in ASD. In contrast, ASD showed reduced activation in the parahippocampal gyrus, involved in scene recognition.
\end{abstract}

Conclusions: When performing a virtual shopping task matching the performance of controls, ASD adolescents hyperactivate three core networks: executive, saliency and social cognition. Parahippocampal hypoactivation is consistent with effortless eidetic scene processing, in line with the notion of peaks and valleys of neural recruitment in individuals with ASD. These hyperactivation/hypoactivation patterns in daily life tasks provide a circuit-level signature of neural diversity in ASD, a possible intervention target.

Keywords: Autism spectrum disorder, Central executive network, Saliency network, Social cognition network, fMRl, Ecological task

\section{*Correspondence: mcbranco@fmed.uc.pt}

${ }^{\dagger}$ Guiomar Oliveira and Miguel Castelo-Branco contributed equally to this

work.

${ }^{4}$ Faculty of Medicine, University of Coimbra, Coimbra, Portugal

Full list of author information is available at the end of the article

\section{Background}

Autism spectrum disorder (ASD) is a heterogeneous neurodevelopmental disorder characterized by a varied severity of deficits in social communication and interaction, and repetitive patterns of behaviour and interests [1].

Considering ASD symptoms, two brain circuits have received particular isolated attention: the social brain original author(s) and the source, provide a link to the Creative Commons licence, and indicate if changes were made. The images or other third party material in this article are included in the article's Creative Commons licence, unless indicated otherwise in a credit line to the material. If material is not included in the article's Creative Commons licence and your intended use is not permitted by statutory regulation or exceeds the permitted use, you will need to obtain permission directly from the copyright holder. To view a copy of this licence, visit http://creativecommons.org/licenses/by/4.0/. The Creative Commons Public Domain Dedication waiver (http://creativeco mmons.org/publicdomain/zero/1.0/) applies to the data made available in this article, unless otherwise stated in a credit line to the data. 
and central executive networks (CEN), while the salience network may also deserve further investigation in this context.

The circuit that supports social cognition includes the prefrontal cortex (PFC), amygdala, anterior thalamus, anterior cingulate cortex (ACC), posterior cingulate cortex (PCC), superior temporal sulcus (STS) and temporo-parietal junction (TPJ) [2-6]. Using tasks operationalizing social cognition, some studies reported distinct activation of these regions in individuals with ASD [7-10], combined with structural differences in areas such as STS, insula, fusiform face area and inferior frontal gyrus [10]. There is growing consensus that the impairments in ASD require multiple-circuit levels of analysis $[4,11-13]$.

Although functional investigations of ASD pathophysiology have focused on social cognition, impairments in other behavioural and cognitive domains are present, such as alterations in the relative perceptual salience of social and non-social stimuli. For example, differences in executive functioning have also been reported in individuals with ASD [14-17]. How changes in these cognitive domains relate to social cognition and other impairments remains an open question. These alterations, in particular the impairments in executive functioning (EF), have an early onset, can aggravate with age and persist despite amelioration of other ASD symptoms $[1,18]$.

EF encompasses cognitive skills, including planning, working memory, attention, inhibition, self-monitoring, self-regulation and initiation [19]. These high-level cognitive processes entail the modulation of lower-level processes, enabling flexible adaptive behaviour [20]. In individuals with ASD, EF is impaired early on and is thought to have a significant influence on social cognition abilities and adaptive behaviour and to be major contributor to everyday deficits, disability and absence of social autonomy [15, 21-23].

Functional neuroimaging of EF in ASD suggested the involvement of the dorsolateral prefrontal cortex (DLPFC) [24-26], superior and inferior parietal lobules [26-30] and anterior frontal areas [29]. Distinct results may depend on the task used, contextual demands, group heterogeneity and ASD comorbidities [20,31]. A recent meta-analysis revealed that both (typical neurodevelopment)TD and participants with ASD recruited PFC regions during EF. ASD presented greater activation in $\mathrm{ACC}$ and lesser activation in the inferior parietal lobule (IPL), left middle frontal gyrus and left medial prefrontal cortex (MPFC) [32].

ASD has also been linked to alterations in the salience network [33], although evidence is more limited. This circuit is involved in selecting the attentional focus, playing a role in switching between internally (for example, the default mode network) and externally focused processing nodes (for example the central executive network) [34-37].

Most of the neuroimaging studies of the salience network in individuals with ASD focused on the restingstate functional connectivity and showed inconsistent results [11,38-40]. It has been proposed that ASD and TD participants can be discriminated based on hyperconnectivity within the salience network [41]. However, little is known about how this altered resting-state connectivity relates to brain activity during information processing [42]. Task-based experimental designs are therefore needed.

Furthermore, it is still poorly understood how the executive network interrelates with the salience network in health and disease and how it functionally connects with social networks. For example, TPJ, which is related to the theory of mind and the distinction between self and other, is also associated with the direction of attention for salient cues.

Cognitively demanding goal-directed tasks in the human brain are thought to involve the dynamic interplay of these large-scale neural networks, in particular the salience network and the CEN [43]. This raises the question how individuals with ASD recruit these networks when attempting to successfully perform real-life tasks.

In this study, we investigated task-driven group differences with a focus on EF and its connected networks. We expected higher activity in EF networks, posing a challenge to the other networks and in particular, the saliency hubs because of their switching role between task-positive and other networks. To achieve this goal, we used an fMRI design to study brain activity of ASD and TD participants while performing a task that required strong executive function demands. Cue saliency processing in the context of a virtual ecological social situation was also a feature of this goal-oriented task. We hypothesized that this approach would show the group differences in central executive network and its concomitant impact on related salience and social brain networks.

We recorded fMRI data while participants performed a task developed at our Lab: the EcoSupermarketX [44], a virtual reality task, monitored with eye-tracking, consisting of going shopping to a supermarket, with three types of sub-tasks that include social cues, non-social cues, or no cues. EcoSupermarketX was based in two main premises: on the one hand, shopping is a good example of a real-world task that often draws heavily on EF, contextual cueing and social/non-social cognition, and on the other hand, different assessment and rehabilitation studies of 
ASD populations have successfully used supermarket settings $[45,46]$.

\section{Methods \\ Participants}

A total of 34 participants took part in the study: 18 individuals with ASD without intellectual disability and 16 TD controls. Due to exclusion criteria concerning excessive head movement during the fMRI acquisition, three participants with ASD and two with TD were excluded from the analysis. As a result, 15 individuals with ASD (14 male and 1 female; median age $=16$ years 4 months) and 14 chronological age-matched control participants (12 male and 2 female; median age $=15$ years 2 months) were included for the final analysis.

The participants with ASD were recruited from the Unit of Neurodevelopment and Autism, from the Child Developmental Centre of the Paediatric Hospital of Coimbra. The selection was based on chronological age ( $\geq 10$ years) and on the ability to cooperate in the fMRI acquisition.

ASD diagnosis was assigned on the basis of the gold standard instruments: parental or caregiver interviewAutism Diagnostic Interview-Revised, ADI-R [44, 47], direct structured proband assessment-Autism Diagnostic Observation Schedule, ADOS [48, 49], and clinical examination performed by an experienced neurodevelopmental Paediatrician, based on the current diagnostic criteria for ASD from the Diagnostic and Statistical Manual of Mental Disorders 5, DSM-5 [1]. All participants with ASD had positive results in the ADI-R and ADOS for autism or ASD and met the criteria for ASD from the DSM-5. A comprehensive medical observation excluded associated medical conditions such as epilepsy, neurocutaneous or other genetic syndromes or other usual comorbidities in ASD samples, such as Attention Deficit Hyperactivity Disorder or intellectual disability.

The TD group included 14 participants with typical neurodevelopment who were matched for chronological age, performance intelligence quotient [50], sex and handedness with the ASD group (Mann-Whitney $U$ or Pearson's chi-square test, $p>$.05). The Social Communication Questionnaire which is a screening test for ASD symptoms was completed by the TD group participants' parents to exclude ASD [51]. The positive cut-off for ASD is equal to or above 15 and all participants scored below.

One participant with ASD and one TD participant demonstrated left-hand dominance as it was measured using the Edinburgh Inventory [52]. When necessary, correction to normal vision was ensured using specific eyeglasses compatible with the magnetic field. Given that some individuals with ASD exhibited hypersensitivity to the sound, we worked with each patient, so they were previously familiarized with the magnetic resonance imaging (MRI) sounds and were able to attend and perform experimental tasks inside the scanner. Nonetheless, all participants used hearing protection.

Both groups underwent a comprehensive neuropsychological evaluation and an assessment of the intelligence quotient (IQ) to exclude Intellectual disability (full-scale IQ > 70). All participants included in the study received the Portuguese adapted version of the Wechsler Intelligence Scale for Children-third edition (WISC-III) [53] or the Wechsler Adult Intelligence Scale-third edition (WAIS-III) [54], according to the participant's age.

The demographic characterization of both groups is summarized in Table 1.

\section{Procedure}

The acquisition session comprised one structural sequence and three functional sequences. The EcoSupermarketX paradigm was presented on an LCD monitor $(48.5 \times 87.8 \mathrm{~cm}, 1920 \times 1080$-pixel resolution, $60-\mathrm{Hz}$ refresh rate) which the participants viewed through a mirror mounted above their eyes. The participants could actively navigate the scenario and select the response using a magnetic resonance-compatible joystick (Hybridmojo, San Mateo CA, USA). During the acquisition session, eye-tracking data (sample frequency of $1 \mathrm{~K}$ ) were recorded inside the scanner using Eyelink 1000 software (EyeLink 1000 Plus, SR Research, Mississauga, Ontario, Canada).

\section{EcoSupermarketX}

EcoSupermarketX is a non-immersive virtual reality task that aims to accurately evaluate the social cognition and EF abilities of the participants using a realistic quotidian scenario-a computer-generated supermarket (for the original version of the task, please see our previous work [55]). EcoSupermarketX was generated with Vizard Virtual Reality toolkit 5.2 (WorldViz, Santa Barbara, USA).

The task in the MRI was preceded by a similar task outside the scanner [55], so the participants were already familiarized with the stimuli. The task has a joystick that allowed the participants to navigate in the scenario and to rotate (as if they were turning the head and looking right or left).

The experimental task consisted of three separate runs with variable duration (since it was dependent on the individual performance). At the end, a total of 18 trials (grocery list presentation + shopping) were performed. Each trial started with the grocery list (three items) presented as an instruction on a trial-by-trial basis: "Find strawberry cake" followed by "Find sausages" and then "Find olive oil", for example (each item image and name 
Table 1 Characterization of the ASD and TD groups

\begin{tabular}{|c|c|c|c|}
\hline & ASD & TD & \\
\hline & $\begin{array}{l}\text { Median } \\
\text { (IQR; min-max) }\end{array}$ & $\begin{array}{l}\text { Median } \\
\text { (IQR; min-max) }\end{array}$ & \\
\hline $\mathbf{N}$ & 15 & 14 & \\
\hline Sex (M/F) & $14 / 1$ & $12 / 2$ & $p=.501^{*}$ \\
\hline CA (years and months) & $\begin{array}{l}16 \text { y } 5 \mathrm{~m} \\
(4 \text { y } 5 \mathrm{~m} ; 12 \text { y } 2 \mathrm{~m}-22 \text { y } 4 \mathrm{~m})\end{array}$ & $\begin{array}{l}15 \text { y } 9 \mathrm{~m} \\
(2 \text { y } 8 \mathrm{~m} ; 10 \text { y } 8 \mathrm{~m}-18 \text { y } 6 \mathrm{~m})\end{array}$ & $p=.290^{*}$ \\
\hline Handedness (R/L) & $14 / 1$ & $13 / 1$ & $p=.960^{*}$ \\
\hline FSIQ & $\begin{array}{l}100.0 \\
(24 ; 71-137)\end{array}$ & $\begin{array}{l}116.5 \\
(24 ; 92-152)\end{array}$ & $p=.004$ \\
\hline VIQ & $\begin{array}{l}97.0 \\
(17 ; 78-126)\end{array}$ & $\begin{array}{l}120.5 \\
(29 ; 98-145)\end{array}$ & $p<.001$ \\
\hline PIQ & $\begin{array}{l}101.0 \\
(35 ; 73-136)\end{array}$ & $\begin{array}{l}107.0 \\
(21 ; 85-146)\end{array}$ & $p=.172^{*}$ \\
\hline ADI-R RSI & $\begin{array}{l}16.0 \\
(11 ; 7-26)\end{array}$ & - & \\
\hline ADI-R L/C & $\begin{array}{l}10.0 \\
(4 ; 3-22)\end{array}$ & - & \\
\hline ADI-R RB/I & $\begin{array}{l}4.0 \\
(2 ; 2-11)\end{array}$ & - & \\
\hline ADOS COM & $\begin{array}{l}5.0 \\
(2 ; 3-7)\end{array}$ & - & \\
\hline ADOS SI & $\begin{array}{l}8 \\
(3 ; 4-14)\end{array}$ & - & \\
\hline ADOS Total & $\begin{array}{l}12.0 \\
(5 ; 8-19)\end{array}$ & - & \\
\hline
\end{tabular}

Note. $A S D$, autism spectrum disorder group; $T D$, typical neurodevelopment group; $I Q R$, interquartile range; min, minimum; $m a x$, maximum; $M$, male; $F$, female; $C A$, chronological age; $R$, right; $L$, left; FSIQ, full-scale intelligence quotient (IQ); VIQ, verbal IQ; PIQ, performance IQ; $A D I-R R S I, A D I-R$ Reciprocal Social Interactions; $A D I-R$ $L / C$, Autism Diagnostic Interview-Revised Language/Communication; ADI-R RB/I, ADI-R Repetitive Behaviours/Interests; ADOS COM, ADOS Communication; ADOS SI, ADOS Social Interaction. *Mann-Whitney $U$ or Pearson's chi-square $p>.05$

appeared for $3 \mathrm{~s}$, see Fig. 1). Then, the participant was instructed to search and pick those groceries from the supermarket shelves. The groceries were replaced randomly in the shelves on a trial-by-trial basis. For every single list with three items, which defines a trial, the participant had a maximum of $3 \mathrm{~min}$ to find the groceries and conclude the "shopping". Nevertheless, the trial ends as the participant completes the list. Participants were instructed to collect all items in the sequence they appeared in the list, and as fast and accurately as possible. They had to plan and monitor their behaviour to complete the task successfully.

Additionally, participants were informed that during the task three situations could happen: they could have help from a person (an avatar) - social cue; an arrownon-social cue; or no help at all-no cue. They were not told what specifically the person (avatar gazing to the grocery) was doing or what kind of arrows (wood arrow) were presented (Fig. 1). Participants performed six trials per cue type. There were $30 \mathrm{~s}$ of baseline (black screen with a central cross) and between the instruction (grocery list encoding) and the beginning of the shopping, there was is a variable interval of 12,14 or $16 \mathrm{~s}$.
To reduce merely memory constraints, an image of the requested item from the list was displayed in the upper right corner after $40 \mathrm{~s}$ of fruitless searching, giving the opportunity to the participant to conclude the trial. Focusing on the enhancement of realism and ecological validity, realistic three-dimensional (3D) forms and commercial brands were used to depict the groceries included in the supermarket scenario.

\section{Eye-tracking recording and measures}

Eye movements were tracked using an infrared-emitting video-based eye tracker (EyeLink 1000 Plus, SR Research, Mississauga, Ontario, Canada). We used mono mode and pupil corneal reflection, at a $1 \mathrm{~K}$ sample rate. The tracker has a reported gaze position accuracy of $0.25-0.50^{\circ}$ and a spatial resolution of 0.05 . A 9-point calibration procedure with a fixation circle was performed before each run. After the calibration, there was a validation trial to ensure the precision of the data collection (tracking error smaller than $1^{\circ}$ visual angle). As participants were performing a dynamic virtual-reality task in which they were navigating around the supermarket, the frames in the screen were always different for all participants. In 


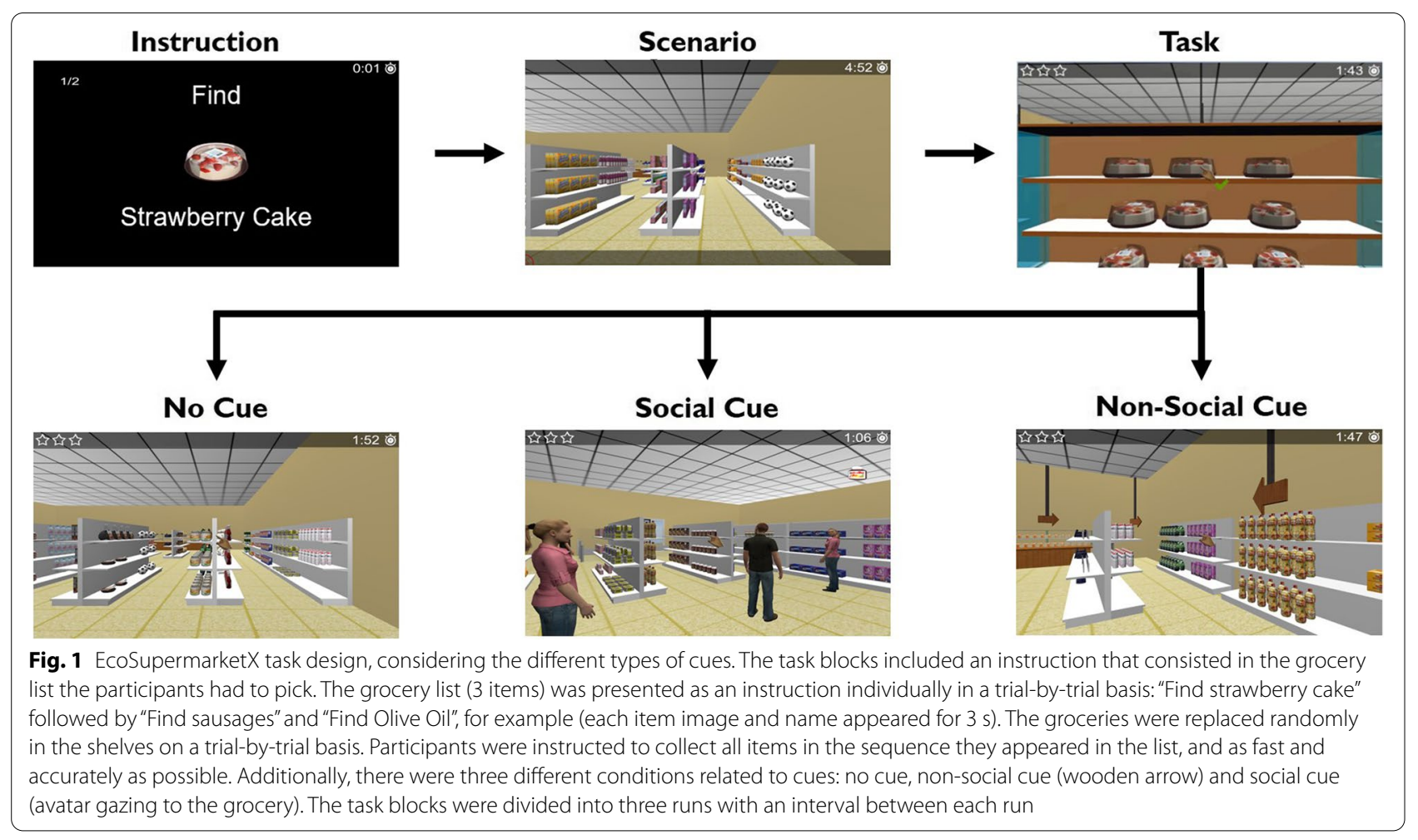

this way, the areas of interest (AOI) were defined in the virtual-reality software, which received the participants' gaze coordinates from the eye-tracking software in a real-time mode. Using those screen coordinates, we computed the time that the participant was looking at each AOI on a real-time basis. The AOI related to the different types of cues were defined: head (because the avatar is looking at the item) and arrow.

\section{fMRI data acquisition and pre-processing}

MRI experiments were performed on a 3 Tesla Magnetom Prisma Fit scanner (Siemens, Erlangen, Germany), using a 64-channel head coil. The scanning session included one T1-weighted 3D anatomical magnetizationprepared rapid acquisition gradient echo pulse sequence, with repetition time $(\mathrm{TR})=2530 \mathrm{~ms}$, echo time $(\mathrm{TE})=$ $3.5 \mathrm{~ms}$, resolution $1 \mathrm{~mm}^{3}$ (voxel size $1.0 \times 1.0 \times 1.0 \mathrm{~mm}$ ), flip angle $=7^{\circ}, 192$ slices, field of view $($ FOV $)=256 \times$ $256 \mathrm{~mm}$ and a slice thickness of $1 \mathrm{~mm}$.

Afterwards, three functional runs were acquired using a $\mathrm{T} 2 \%$-weighted gradient echo-planar imaging sequence, with a slice thickness of $3 \mathrm{~mm}$ and voxel size $3 \mathrm{~mm}^{2}, 37$ interleaved slices without gap, parallel to the AC-PC line, $\mathrm{TR}=2000 \mathrm{~ms}, \mathrm{TE}=30 \mathrm{~ms}$, fip angle of $75^{\circ}$ and FOV of $210 \times 210$. On average, the scanning session lasted 45 min.
Data pre-processing was performed on BrainVoyager 21.4 software (Brain Innovation, Maastricht, The Netherlands). Pre-processing included slice-scan time correction, 3D head-motion correction and temporal high-pass filtering ( 2 cycles per run). Participants exceeding $6 \mathrm{~mm}$ of movement in any axis were excluded from further analysis ( $n=5$; 3 ASD and 2 TD). For the rest of the participants, we used motion as a confound predictor in the GLM model during the pre-processing phase. Data were normalized to a Montreal Neurological Institute (MNI) space and spatially smoothed using a Gaussian kernel with FWHM of $6 \mathrm{~mm}$.

\section{Data Analysis}

All statistical analysis was completed with the support of the Statistical Package for Social Sciences, version 26 (SPSS ${ }^{\circledR}$, Chicago, IL, USA) and the Brain Voyager 21.4 software (Brain Innovation, Maastricht, the Netherlands).

\section{Behavioural EcoSupermarketX data analysis}

Several parameters were defined for the analysis of the EcoSupermarketX performance. Information about the $\mathrm{EF}$ that, in our perspective, is reflected by each EcoSupermarketX behavioural measure was extracted. The different behavioural measures/parameters defined were item errors, sequencing errors, time, distance and head rotation, which are detailed below: 
Item errors. The number of picked items of the EcoSupermarketX scenario that were not in the list of groceries divided by the number of items in the grocery list $\times 100$ (e.g. select a toy, when the toy was not in the list).

Sequencing errors. The number of picked items of the EcoSupermarketX scenario that were in the incorrect sequence in the list of groceries divided by the number of items in the grocery list $\times 100$ (e.g. to select sausages before cereals, when the cereals were first in the list).

Total time. Performance time (in seconds) - The time the participant was engaged in the execution of the task: looking for and grabbing the products that were in the grocery list (time elapsed from the end of the grocery list memorization to the last picked item).

Total distance. Performance distance-The distance the participant goes through in the execution of the task: looking for and grabbing of the products that are in the grocery list).

Head rotation (orienting response). Sum of virtual head rotations by the participant (in degrees) during the time of execution of the task. This parameter aims to reflect attentional control, psychomotor and processing speed, planning and motor time.

Nonparametric statistics (Mann-Whitney $U$ tests) were carried out for all statistical analyses to avoid biases due to deviations from normality and variance heterogeneity.

\section{Functional magnetic resonance data analysis}

Statistical analysis was performed at the group level. For the GLM approach, the predictor's model was obtained by convolution of the boxcar time course of each condition (no cue, non-social cue, social cue) with a two-gamma hemodynamic response function. The individual block duration was defined up to the participant's response.

First, to compute a whole-brain statistical maps for group effect, an overlay random-effects (RFX) ANOVA with within-factors "Cue" (no cue, non-social cue, social cue) and between-factor "group" (ASD vs. TD) was conducted. These statistical maps were corrected for multiple comparisons using a cluster threshold method with a fixed $p$-value of .05 and an estimated minimum cluster extension of 70 contiguous voxels. The extension was estimated using Monte Carlo simulations (1000 iterations). This map revealed areas for which there are fundamental differences between groups irrespective of other factors.
In addition, for each identified region, post hoc $t$-tests were computed whenever significant group effects were found.

\section{Ethics statement}

All the procedures were approved by the ethics committees from our University and Hospital Centre of Coimbra and were conducted in accordance with the Helsinki declaration. Written informed consent was obtained from the parents/guardians of all participants or, when appropriate, the participants themselves. Children and adolescents also gave oral informed consent.

\section{Community involvement}

The community of local Patient Associations is involved in this study.

\section{Results}

\section{Behavioural results}

Behavioural analysis revealed that ASD and TD groups achieved similar performances in all EcoSupermarketX task parameters $(p>.05$, Mann-Whitney $U)$, except for the items' total time and distance in the social cue condition. The total time taken to perform the task was significantly higher for the ASD group (median [Mdn] = 34.5) than the TD group ( $M d n=26.9), U=58.00, p$ $=.041, d=.824$. The same pattern was present in the total distance, with the ASD group $(\mathrm{Mdn}=50.1)$ walking longer distances in the social cue condition than the TD group ( $\mathrm{Mdn}=43.0$ ), $U=44.00, p=.007, d=$ 1.137. These results are summarized in Fig. 2.

Group performances were matched, since no significant statistical differences were seen, in other measured parameters: item errors, total sequencing errors and head rotation, both in social cue, non-social and no cue conditions.

\section{Eye-tracking measures}

The time looking at the different AOIs of social or nonsocial relevance (head or arrow) that were related to the different types of cues (social and non-social, respectively) was compared between the two groups (ASD and TD).

A Mann-Whitney $U$ test indicated no differences between the ASD and TD groups in the AOIs head ( $U$ $=99.00, p=1.000)$ and Arrow $(U=114.00, p=.482)$.

\section{fMRI: whole-brain analysis of between-group effects in EcoSupermarketX task}

The whole-brain RFX ANOVA revealed significant group effects in prefrontal (including ventromedial, 

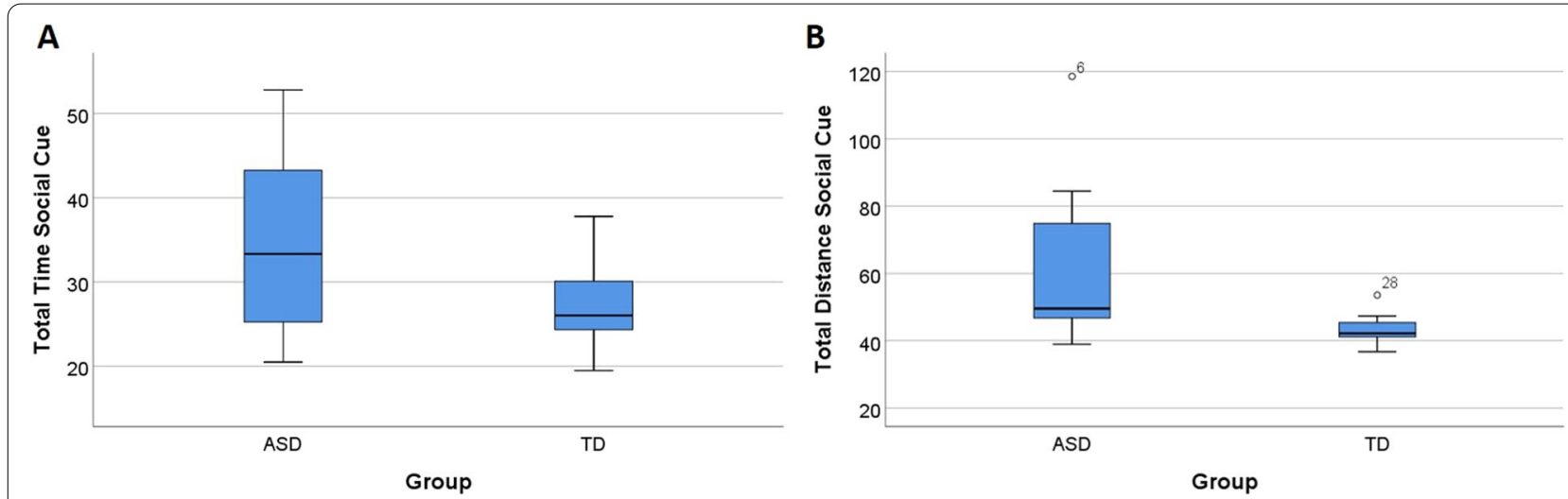

Fig. 2 Performance across time and distance in social cue condition in ASD and TD groups. A Total time for ASD and TD groups for the social cue condition. B Total distance for ASD and TD groups for the social cue condition. Boxplots: central mark —-median; edges of box - 25th and 75th percentiles; whiskers - most extreme data points (minimum and maximum). Note. ASD, autism spectrum disorder, TD, typical neurodevelopment.

orbitofrontal and anterior cortex), temporal (in particular the TPJ and the temporal pole) and visual areas. The differences between the pattern observed in ASD and TD groups are detailed in Table $2(F \geq 4.21 ; p<.05$, corrected for multiple comparisons) and further highlighted in Fig. 3.

Region of interest-based post hoc $t$-tests confirmed that across social cognition, executive and saliency networks ASD showed higher BOLD activity when compared with TD in response to EcoSupermarketX task conditions. This included regions such as the middle frontal gyrus, ventrolateral $\mathrm{PFC}$, ventromedial $\mathrm{PFC}$, orbitofrontal cortex (OFC), inferior frontal gyrus, middle temporal cortex, temporal pole, TPJ, ACC and supramarginal gyrus. The same analysis revealed higher BOLD activity for TD compared to ASD only in the parahippocampal gyrus (for further details on statistical significance, see Table 2).

\section{Discussion}

We investigated the neural correlates of executive functioning when ASD and healthy participants engage in a demanding, goal-oriented ecological task, where social and non-social cues are present. We used markers of EF under distinct task constraints, with explicit manipulations of the types of cues that improve task performance. We also monitored visual attention to specific AOI's social (avatar's head) and non-social (arrow) cues, while shopping.

\section{Preserved ASD performance for non-social conditions}

We found that, in the absence of a cue or with the nonsocial cue, groups did not differ. ASD group only performed worse in two specific behavioural measures of the social cue condition: total time and distance taken to do the task. In fact, participants with ASD did not differ from TD in item errors, sequencing errors and total head rotation in all conditions, corroborating the notion that groups were overall matched in terms of performance.

\section{Higher ASD recruitment of executive, saliency and social networks during EcoSupermarketX}

When performing this virtual ecological daily living task-going shopping, we found differential activation across three main networks: social, executive, and saliency circuits. Accordingly, we found higher activity by the ASD group when compared to TD in areas that are pivotal in social brain circuits, namely the TPJ, ventromedial PFC and inferior parietal lobule. Importantly, the TPJ, a part of the ventral attention system, has also been implicated in social and emotional processing [56-59], including joint attention [60-62], attentional reorienting to salient cues [63] and visually triggered shifts of attention that share common neural substrates with gaze perception [64]. Our task had three blocks of increasing executive load and incorporated social and non-social cues, with different degrees of saliency, which is ideally suited to recruit attentional, executive and saliency networks. These aspects are quite important in our task design and are related to core characteristics of individuals with ASD, as corroborated by our previous behavioural study [55]. Moreover, it is also known that the TPJ is most reliably activated when participants engage in tasks involving the inference of goals or end states for described actions [65].

Larger recruitment of areas of the executive network was also found in individuals with ASD during EcoSupermarketX, namely in the middle frontal gyrus, and also pregenual ACC which is part of the salience network. 


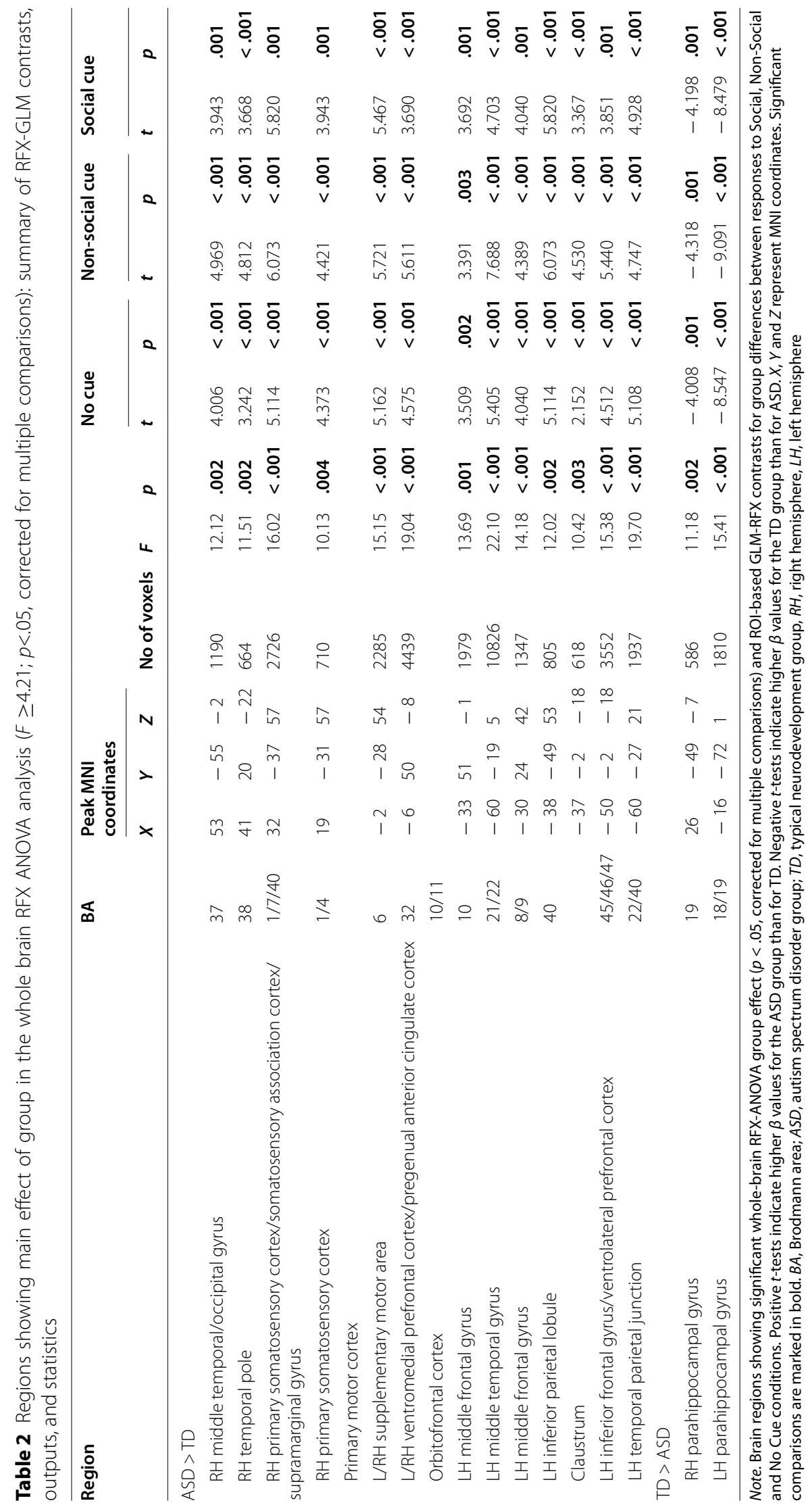




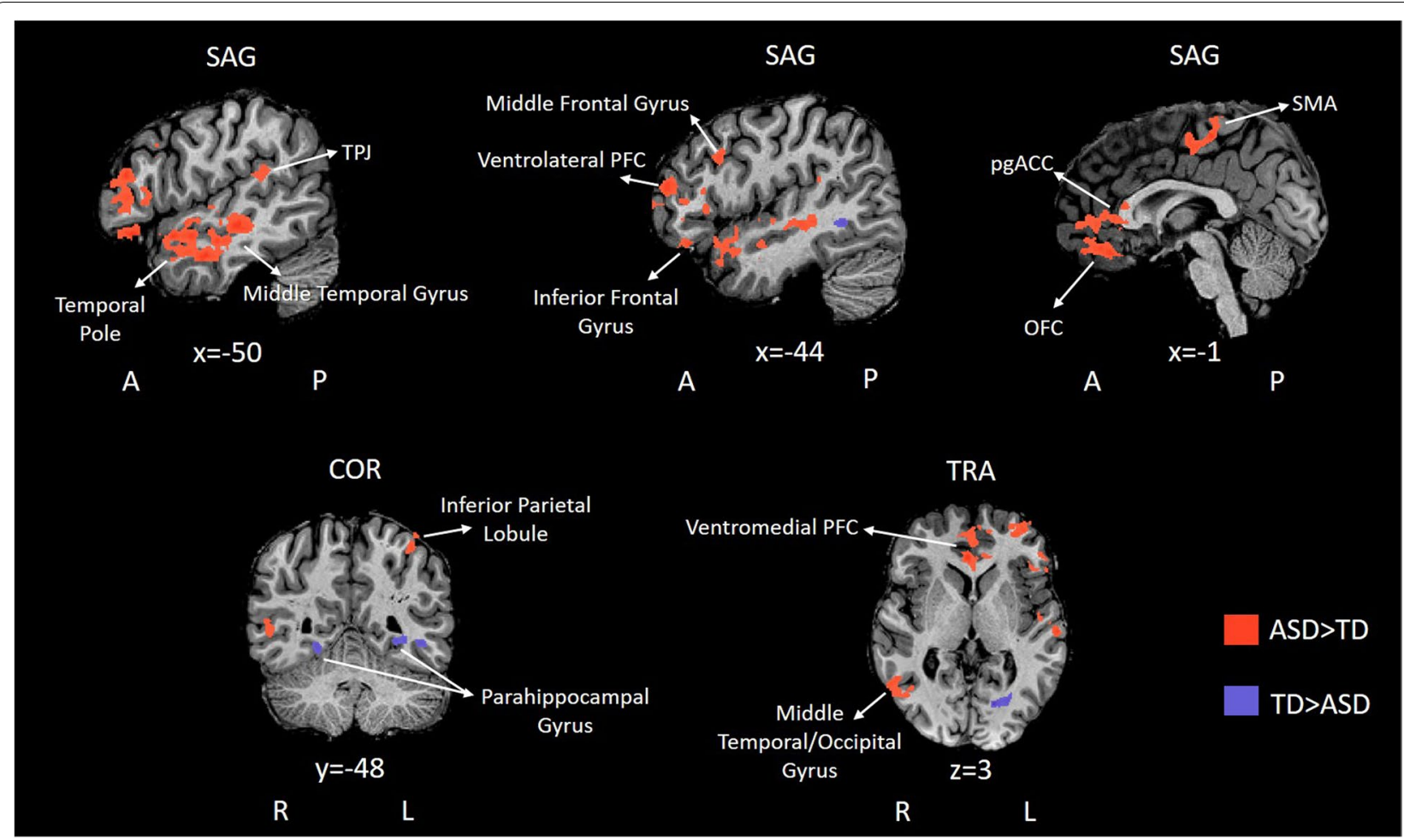

Fig. 3 RFX ANOVA group effects for EcoSupermarketX task. Statistical maps from group analysis overlaid on sagittal, coronal, and transversal slices of a representative subject. Red clusters depict regions where BOLD activity was higher for individuals with ASD than TD. Blue clusters depict regions where BOLD activity was lower for individuals with ASD than TD. Slice locations are given in MNI coordinates. NOTE. ASD, autism spectrum disorder group, TD, typical neurodevelopment group, TPJ, temporal-parietal junction, PFC, prefrontal cortex, pgACC, pregenual anterior cingulate cortex; OFC, orbitofrontal cortex, SMA, supplementary motor area, A, anterior; P, posterior, R, right, L, left; SAG, sagittal plane; COR, coronal plane; TRA, transversal plane; MNI, Montreal Neurological Institute

The only region that showed reduced activity in individuals with ASD as compared to controls was the parahippocampal gyrus. It is known to be involved in scene recognition, spatial navigation and memory encoding and retrieval [66], which are required to perform the presented task and it is known to be a strength in the cognitive profile of individuals with ASD.

We interpret our results in light of the notion that the over-recruitment observed for the three main networks reflects the large cognitive burden that this type of ecological task poses to participants with ASD. Given that individuals with ASD are quite functional in terms of scene recognition and navigation, this might explain why parahippocampal under-recruitment occurs in comparison to controls. In other words, relative proficiency leads to less brain activation because fewer resources are needed. A study comparing TD adolescent girls before and after practice on a visual-spatial problem-solving computer game, Tetris, showed that brain activity decreases after practice [67]. Additionally, a recent study [68] showed that no impairments emerged in visuospatial working memory in individuals with ASD, as compared with TD individuals, which corroborates our previous work [69], showing that this ability is relatively spared. This is in line with the notion of peaks and valleys of performance in individuals with ASD.

Importantly, this pattern of simultaneous recruitment of three major networks (and lower activity for the parahippocampal gyrus) was found for the three conditions in the task. As the final performance of the task is quite similar between the groups, we may speculate that the ASD group maintains a higher neural effort, as a putative compensatory mechanism, to reach similar performance levels as the TD group. Although neural compensation mechanisms are still controversial, this neurobehavioural experiment showed that ASD correctly used the structured cues as TD does in goal-oriented tasks.

Considering that shopping is a challenging task for the majority of individuals with ASD and our results showed they are engaged in an extra mental effort in the executive, saliency and social brain networks to achieve accurate performance; we may assume that they had to make a more focused effort, at the attentional, executive and 
emotional levels than the TD group. This would explain why there is higher activity in the OFC, an area that is part of the limbic system, being involved in the control emotional reactions in certain social situations, as well as the process of decision-making and self-control [70]. OFC has also been thought to contain a representation of task space, which is especially critical for behaviour when states are unobservable from sensory input [71].

Similar performance scores and different patterns of brain activation relative to controls had been reported in functional neuroimaging studies but with distinct significance: these studies focused on response inhibition in adults with ASD [28, 72, 73]. Kana et al. [72] found that the adults with ASD relative to controls had decreased activity, namely the in ACC, while Schmitz et al. [28] found increased activity for the ASD group in several brain regions known to be involved in response inhibition, including the inferior frontal gyrus and the OFC during a simple Go/NoGo task using non-social stimuli. However, most of these studies differ from ours in two very important aspects: ecologic setup and multiple task demands (social, attentional, executive) that are inherent to our paradigm.

Our non-intellectually disabled individuals with ASD understood and performed the shopping task as the TD group and presented little performance differences and no changes in the eye-tracking measures when compared to the TD group. Our participants with ASD used the structured cues as TD in a goal-oriented manner.

Concerning the limitations of our study, they are reflected in its small sample size.

\section{Conclusions}

In conclusion, our functional imaging results are consistent with the notion that hyperactivation of particular networks may provide a compensatory mechanism to preserve performance [73] in contrast with scene recognition parahippocampal networks. It is remarkable that for the first time in the current study this was observed simultaneously across executive, saliency and social cognition networks, which reinforces the dysfunction of this pivotal pathway in individuals with ASD, a network signature that may represent a possible intervention target.

\section{Abbreviations}

3D: Three-dimensional; A: Anterior; ACC: Anterior cingulate cortex; ADI-R: Autism Diagnostic Interview—Revised; ADI-R L/C: ADI-R Language/Communication; ADI-R RB/I: ADI-R Repetitive Behaviours/Interests; ADI-R RSI: ADI-R Reciprocal Social Interactions; ADOS: Autism Diagnostic Observation Schedule; ADOS COM: ADOS Communication; ADOS SI: ADOS Social Interaction; AOI: Area of interest; ASD: Autism spectrum disorder; BA: Brodmann area; BOLD: Blood-oxygen-level-dependent; CA: Chronological age; CEN: Central executive network; COR: Coronal plane; DLPFC: Dorsolateral prefrontal cortex; DSM-5: Diagnostic and Statistical Manual of Mental Disorders 5; EF: Executive functioning; EPI: Echo-planar imaging; F: Female; fMRI: Functional magnetic resonance imaging; FOV: Field of view; FSIQ: Full-scale intelligence quotient; GLM: General linear model; IPL: Inferior parietal lobule; IQ: Intelligence quotient; IQR: Interquartile range; L: Left; LCD: Liquid crystal display; LH: Left hemisphere; M: Male; Mdn: Median; MFG: Middle frontal gyrus; MNI: Montreal Neurological Institute; MPFC: Medial prefrontal cortex; MPRAGE: Magnetization-prepared rapid acquisition gradient echo; MRI: Magnetic resonance imaging; OFC: Orbitofrontal cortex; P: Posterior; pgACC: Pregenual anterior cingulate cortex; PCC: Posterior cingulate cortex; PFC: Prefrontal cortex; PHG: Parahippocampal gyrus; PIQ: Performance intelligence quotient; R: Right; RH: Right hemisphere; RFX: Random-effects; SAG: Sagittal plane; SMA: Supplementary motor area; SPL: Superior parietal lobule; SPSS: Statistical Package for Social Sciences; STS: Superior temporal sulcus; TD: Typical neurodevelopment; TE: Echo Time; TPJ: Temporo-parietal junction; TR: Repetition time; TRA :Transversal plane; VIQ: Verbal intelligence quotient; vIPFC: Ventrolateral prefrontal cortex; vmPFC: Ventromedial prefrontal cortex; WAIS-III: Wechsler adult intelligence scale-Third Edition; WISC-III: Wechsler intelligence scale for children-Third Edition.

\section{Acknowledgements}

The authors thank all participants and their families for their willingness to participate in this study, particularly the participants with autism spectrum disorder. The authors would like to acknowledge Alexandre Malhão and Hélio Gonçalves for their contributions to EcoSupermarketX task.

\section{Authors' contributions}

Conceived and designed the study: Susana Mouga, Guiomar Oliveira and Miguel Castelo-Branco. Programmed the task: Isabel Catarina Duarte. Performed the study: Susana Mouga. Contributed with data collection: Susana Mouga, Cátia Café, Daniela Sousa, Frederico Duque. Analysed the data: Susana Mouga and Isabel Catarina Duarte. Supervision: Guiomar Oliveira and Miguel Castelo-Branco. Writing - original manuscript preparation: Susana Mouga. Writing — review and editing: Isabel Catarina Duarte, Guiomar Oliveira and Miguel Castelo-Branco. All authors read and approved the final manuscript.

\section{Funding}

This research was supported by the Portuguese Foundation for Science and Technology: PAC MEDPERSYST POCI-01-0145- FEDER-016428, CONNECT-BCI, POCI-01-0145-FEDER-30852, FCT/UID/4950B/2020, UID- P-4950, DSAIPA/ DS/0041/2020, European Commission (AIMS-2-Trials), individual PhD scholarship: SFRH/BD/102779/2014 to SM, CENTRO-01-0145-FEDER-000016 to SM and DS (PI: MCB) and Luso-American Development Foundation (FLAD) Life Sciences. The funders had no role in study design, data collection and analysis, decision to publish or preparation of the manuscript.

\section{Availability of data and materials}

The datasets used and/or analysed during the current study are available on reasonable request.

\section{Declarations}

\section{Ethics approval and consent to participate}

All the procedures were approved by the ethics committees from the Faculty of Medicine from Coimbra University (CE-11/2013) and Centro Hospitalar e Universitário de Coimbra (CHUC-102-13) and were conducted in accordance with the Helsinki declaration. Written informed consent was obtained from the parents/guardians of all participants or, when appropriate, the participants themselves. Children and adolescents also gave oral informed consent.

\section{Consent for publication}

Not applicable.

\section{Competing interests}

The authors have declared that no competing interests exist.

\section{Author details}

${ }^{1}$ CIBIT - Coimbra Institute for Biomedical Imaging and Translational Research, University of Coimbra, Azinhaga de Santa Comba, 3000-548 Coimbra, Portugal. ${ }^{2}$ ICNAS - Institute of Nuclear Sciences Applied to Health, University of Coimbra, Coimbra, Portugal. ${ }^{3}$ Neurodevelopmental and Autism Unit 
from Child Developmental Center, Hospital Pediátrico, Centro Hospitalar e Universitário de Coimbra, Coimbra, Portugal. ${ }^{4}$ Faculty of Medicine, University of Coimbra, Coimbra, Portugal.

Received: 2 September 2021 Accepted: 10 January 2022 Published online: 25 January 2022

\section{References}

1. American Psychiatric Association. Diagnostic and Statistical Manual of Mental Disorders. 5th ed. Arlington: American Psychiatric Publishing; 2013.

2. Kennedy DP, Adolphs R. The social brain in psychiatric and neurological disorders. Trends Cogn Sci [Internet]. 2012;16(11):559-72 Available from: https://www.ncbi.nlm.nih.gov/pmc/articles/PMC3624763/pdf/nihms 412728.pdf.

3. Arioli M, Canessa N. Neural processing of social interaction: coordinatebased meta-analytic evidence from human neuroimaging studies. Hum Brain Mapp. 2019;40(13):3712-37.

4. Müller RA, Fishman I. Brain connectivity and neuroimaging of social networks in autism. Trends Cogn Sci. 2018;22(12):1103-16. Available from. https://doi.org/10.1016/j.tics.2018.09.008.

5. Schurz M, Radua J, Aichhorn M, Richlan F, Perner J. Fractionating theory of mind: A meta-analysis of functional brainimaging studies. Neurosci Biobehav Rev. 2014:42:9-34.

6. Wolf I, Dziobek I, Heekeren HR. Neural correlates of social cognition in naturalistic settings: A model-free analysis approach. Neuroimage. 2010:49(1):894-904

7. Kana RK, Libero LE, Hu CP, Deshpande HD, Colburn JS. Functional Brain Networks and White Matter Underlying Theoryof-Mind in Autism. Soc Cogn Affect Neurosci. 2014;9(1):98-105.

8. White SJ, Frith U, Rellecke J, Al-Noor Z, Gilbert SJ. Autistic adolescents show atypical activation of the brain's mentalizing system even without a prior history of mentalizing problems. Neuropsychologia. 2014;56:17-25.

9. Kim E, Kyeong S, Cheon K-A, Park B, Oh M-K, Chun JW, et al. Neural responses to affective and cognitive theory of mind in children and adolescents with autism spectrum disorder. Neurosci Lett. 2016;621:117-25.

10. Patriquin MA, DeRamus T, Libero LE, Laird A, Kana RK. Neuroanatomical and neurofunctional markers of social cognition in autism spectrum disorder. Hum Brain Mapp. 2016;37(11):3957-78.

11. Chen H, Uddin LQ, Duan X, Zheng J, Long Z, Zhang Y, et al. Shared atypical default mode and salience network functional connectivity between autism and schizophrenia. Autism Res. 2017;10(11):1776-86.

12. Eack SM, Wojtalik JA, Keshavan MS, Minshew NJ. Social-cognitive brain function and connectivity during visual perspective-taking in autism and schizophrenia. Schizophr Res. 2017;183:102-9.

13. Park MTM, Raznahan A, Shaw P, Gogtay N, Lerch JP, Chakravarty MM. Neuroanatomical phenotypes in mental illness: identifying convergent and divergent cortical phenotypes across autism, ADHD and schizophrenia. J Psychiatry Neurosci. 2018;43(3):201-12.

14. Chita-Tegmark M. Social attention in ASD: a review and meta-analysis of eye-tracking studies. Res Dev Disabil [Internet]. 2016;48:79-93 Available from: https://linkinghub.elsevier.com/retrieve/pii/S0891422215001821.

15. Lai CLE, Lau Z, Lui SSY, Lok E, Tam V, Chan Q, et al. Meta-analysis of neuropsychological measures of executive functioning in children and adolescents with high-functioning autism spectrum disorder. Autism Res. 2017;10(5):911-39. Available from. https://doi.org/10.1002/aur.1723.

16. Lai M-C, Lombardo MV Baron-Cohen S. Autism. Lancet. 2014;383(9920):896-910 Available from: https://linkinghub.elsevier.com/ retrieve/pii/S0140673613615391.

17. Ruta L, Famà Fl, Bernava GM, Leonardi E, Tartarisco G, Falzone A, et al. Reduced preference for social rewards in a novel tablet based task in young children with Autism Spectrum Disorders. Sci Rep. 2017;7(1):3329.

18. Mouga S, Correia BR, Café C, Duque F, Oliveira G. Language predictors in autism spectrum disorder: insights from neurodevelopmental profile in a longitudinal perspective. J Abnorm Child Psychol. 2020;48(1):149-61 Available from: http://link.springer.com/10.1007/s10802-019-00578-7.

19. Goldstein S, Naglieri JA. Introduction: a history of executive functioning as a theoretical and clinical construct. In: Goldstein S, Naglieri JA, editors. Handbook of executive functioning [Internet]. New York: Springer New
York; 2014. p. 1-567. Available from: http://link.springer.com/10.1007/ 978-1-4614-8106-5

20. Gilbert SJ, Burgess PW. Executive function. Curr Biol. 2008;18(3):R110-4 Available from: https://linkinghub.elsevier.com/retrieve/pii/B978012809 3245060016.

21. Demetriou EA, Lampit A, Quintana DS, Naismith SL, Song YJC, Pye JE, et al. Autism spectrum disorders: a meta-analysis of executive function. Mol Psychiatry. 2018;23(5):1198-204. Available from:. https://doi.org/10.1038/ mp.2017.75.

22. Geurts HM, van den Bergh SFWM, Ruzzano L. Prepotent response inhibition and interference control in autism spectrum disorders: two metaanalyses. Autism Res. 2014;7(4):407-20.

23. Leung RC, Zakzanis KK. Brief report: Cognitive flexibility in autism spectrum disorders: a quantitative review. J Autism Dev Disord. 2014;44(10):2628-45 Available from: http://link.springer.com/10.1007/ s10803-014-2136-4.

24. Dichter GS, Belger A. Atypical modulation of cognitive control by arousal in autism. Psychiatry Res Neuroimaging. 2008;164(3):185-97 Available from: https://linkinghub.elsevier.com/retrieve/pii/S0925492707002521.

25. Luna B, Minshew NJ, Garver KE, Lazar NA, Thulborn KR, Eddy WF, et al. Neocortical system abnormalities in autism: an fMRI study of spatial working memory. Neurology. 2002;59(6):834-40.

26. Shafritz KM, Dichter GS, Baranek GT, Belger A. The Neural circuitry mediating shifts in behavioral response and cognitive set in autism. Biol Psychiatry. 2008;63(10):974-80.

27. Just MA, Cherkassky VL, Keller TA, Kana RK, Minshew NJ. Functional and anatomical cortical underconnectivity in autism: evidence from an fMR study of an executive function task and corpus callosum morphometry. Cereb Cortex. 2007;17(4):951-61 Available from: https://academic.oup. com/cercor/article-lookup/doi/10.1093/cercor/bhl006.

28. Schmitz N, Rubia K, Daly E, Smith A, Williams S, Murphy DGM. Neural correlates of executive function in autistic spectrum disorders. Biol Psychiatry. 2006:59(1):7-16.

29. Solomon M, Ozonoff SJ, Ursu S, Ravizza S, Cummings N, Ly S, et al. The neural substrates of cognitive control deficits in autism spectrum disorders. Neuropsychologia. 2009:47(12):2515-26.

30. Solomon M, Yoon JH, Ragland JD, Niendam TA, Lesh TA, Fairbrother W, et al. The development of the neural substrates of cognitive control in adolescents with autism spectrum disorders. Biol Psychiatry. 2014;76(5):412-21. Available from:. https://doi.org/10.1016/j.biopsych. 2013.08.036.

31. Minshew NJ, Keller TA. The nature of brain dysfunction in autism: Functional brain imaging studies. Curr Opin Neurol [Internet]. 2010;23(2):12430 Available from: http://www.ncbi.n/m.nih.gov/pubmed/20154614.

32. May KE, Kana RK. Frontoparietal network in executive functioning in autism spectrum disorder. Autism Res. 2020;13(10):1762-77.

33. Monk CS, Peltier SJ, Wiggins JL, Weng S-J, Carrasco M, Risi S, et al. Abnormalities of intrinsic functional connectivity in autism spectrum disorders. Neuroimage. 2009;47(2):764-72

34. Sridharan D, Levitin DJ, Menon V. A critical role for the right frontoinsular cortex in switching between central-executive and default-mode networks. Proc Natl Acad Sci [Internet]. 2008;105(34):12569-74 Available from: http//www pnas.org/cgi/doi/10.1073/pnas.0800005105.

35. Menon V, Uddin LQ. Saliency, switching, attention and control: a network model of insula function. Brain Struct Funct [Internet] 2010;214(5-6):655-67 Available from: http://link.springer.com/10.1007/ s00429-010-0262-0.

36. Seeley WW, Menon V, Schatzberg AF, Keller J, Glover GH, Kenna H, et al. Dissociable Intrinsic Connectivity Networks for Salience Processing and Executive Control. J Neurosci. 2007:27(9):2349-56.

37. Seeley WW. The salience network: a neural system for perceiving and responding to homeostatic demands. J Neurosci. 2019:39(50):9878-82.

38. Elton A, Di Martino A, Hazlett HC, Gao W. Neural Connectivity Evidence for a Categorical-Dimensional Hybrid Model of Autism Spectrum Disorder. Biol Psychiatry. 2016 Jul;80(2):120-8.

39. Uddin LQ. Salience processing and insular cortical function and dysfunction. Nat Rev Neurosci. 2015;16(1):55-61.

40. von dem Hagen EAH, Stoyanova RS, Baron-Cohen S, Calder AJ. Reduced functional connectivity within and between 'social' resting state networks in autism spectrum conditions. Soc Cogn Affect Neurosci. 2013:8(6):694-701. 
41. Uddin LQ, Supekar K, Lynch CJ, Khouzam A, Phillips J, Feinstein C, et al. Salience network-based classification and prediction of symptom severity in children with autism. JAMA Psychiatry. 2013;70(8):869-79.

42. Green SA, Hernandez L, Bookheimer SY, Dapretto M. Salience network connectivity in autism is related to brain and behavioral markers of sensory overresponsivity. J Am Acad Child Adolesc Psychiatry. 2016;55(7):618-626.e1. Available from:. https://doi.org/10.1016/j.jaac. 2016.04.013.

43. Chand GB, Dhamala M. Interactions among the brain default-mode, salience, and central-executive networks during perceptual decision-making of moving dots. Brain Connect [Internet]. 2016;6(3):249-54 Available from: http://www.liebertpub.com/doi/10.1089/brain.2015.0379.

44. Le Couteur A, Lord C, Rutter M. The Autism Diagnostic Interview-Revised (ADI-R). Los Angeles CA Western Psychological Services: Western Psychological Services; 2003.

45. Carr EG, Carlson Jl. Reduction of severe behavior problems in the community using a multicomponent treatment approach. J Appl Behav Anal [Internet]. 1993;26(2):157-72 Available from: http://doi.wiley.com/10. 1901/jaba.1993.26-157.

46. Lamash $\mathrm{L}$, Josman $\mathrm{N}$. A metacognitive intervention model to promote independence among individuals with autism spectrum disorder: implementation on a shopping task in the community. Neuropsychol Rehabil. 2019;0(0):1-22. Available from. https://doi.org/10.1080/09602011.2019. 1682621.

47. Lord C, Rutter M, Le Couteur A. Autism Diagnostic Interview-Revised: a revised version of a diagnostic interview for caregivers of individuals with possible pervasive developmental disorders. J Autism Dev Disord. 1994;24(5):659-85 Available from: http://link.springer.com/10.1007/BF021 72145 .

48. Lord C, Rutter M, Goode S, Heemsbergen J, Jordan H, Mawhood L, et al. Autism diagnostic observation schedule: a standardized observation of communicative and social behavior. J Autism Dev Disord. 1989;19(2):185212 Available from: http://www.ncbi.nlm.nih.gov/entrez/query.fcgi? $\mathrm{cmd}=$ Retrieve\&db=PubMed\&dopt=Citation\&list_uids $=2745388$.

49. Lord C, Rutter M. Autism diagnostic observation schedule-WPS (ADOSWPS). Los Angeles CA Western Psychological. 1999.

50. Jarrold C, Brock J. To match or not to match? Methodological issues in autism-related research. J Autism Dev Disord [Internet]. 2004;34(1):81-6 Available from: http://link.springer.com/10.1023/B:JADD.0000018078. 82542.ab.

51. Rutter M, Bailey A, Lord C. Social Communication Questionnaire. Los Angeles: Western Psychological Services; 2003.

52. Oldfield RC. The assessment and analysis of handedness: The Edinburgh inventory. Neuropsychologia. 1971;9(1):97-113.

53. Wechsler D. Wechsler Intelligence Scale for Children-Third Edition (WISC-III) - Portuguese Version (M. R. Simões, A. M. Rocha, and C. Ferreira). Lisbon: Cegoc-Tea; 2003.

54. Wechsler D. In: Simões MR, Rocha AM, Ferreira C, editors. Third Edit Manual for intelligence scale for adults. Portuguese version. Lisboa: Cegoc-Tea; 2008.

55. Mouga S, Duarte IC, Café C, Sousa D, Duque F, Oliveira G, et al. Attentional cueing and executive deficits revealed by a virtual supermarket task coupled with eye-tracking in autism spectrum Disorder. Front Psychol. 2021;12(August):1-16.

56. Bilek E, Ruf M, Schäfer A, Akdeniz C, Calhoun VD, Schmahl C, et al. Information flow between interacting human brains: Identification, validation, and relationship to social expertise. Proc Natl Acad Sci. 2015:112(16):5207-12.

57. Frith U, Frith CD. Development and neurophysiology of mentalizing Philosophical Transactions of the Royal Society B: Biological Sciences; 2003.

58. Schulte-Rüther M, Greimel E, Markowitsch HJ, Kamp-Becker I, Remschmidt H, Fink GR, et al. Dysfunctions in brain networks supporting empathy: An fMRI study in adults with autism spectrum disorders. Soc Neurosci. 2011;6(1):1-21.

59. Krall SC, Rottschy C, Oberwelland E, Bzdok D, Fox PT, Eickhoff SB, et al. The role of the right temporoparietal junction in attention and social interaction as revealed by ALE meta-analysis. Brain Struct Funct. 2015;220(2):587-604.

60. Oberwelland E, Schilbach L, Barisic I, Krall SC, Vogeley K, Fink GR, et al. Look into my eyes: Investigating joint attention using interactive eye-tracking and fMRI in a developmental sample. Neuroimage. 2016;130:248-60.

61. Redcay E, Dodell-Feder D, Pearrow MJ, Mavros PL, Kleiner M, Gabrieli JDE, et al. Live face-to-face interaction during fMRI: A new tool for social cognitive neuroscience. Neuroimage. 2010 May;50(4):1639-47.

62. Schilbach L, Wilms M, Eickhoff SB, Romanzetti S, Tepest R, Bente G, et al. Minds Made for Sharing: Initiating Joint Attention Recruits Rewardrelated Neurocircuitry. J Cogn Neurosci. 2010;22(12):2702-15.

63. Carter RM, Huettel SA. A nexus model of the temporal-parietal junction. Trends Cogn Sci. 2013;17(7):328-36.

64. Itier RJ, Batty M. Neural bases of eye and gaze processing: The core of social cognition. Neurosci Biobehav Rev. 2009;33(6):843-63.

65. Van Overwalle F. Social cognition and the brain: A meta-analysis. Hum Brain Mapp. 2009;30(3):829-58.

66. Aminoff EM, Kveraga K, Bar M. The role of the parahippocampal cortex in cognition. Trends Cogn Sci. 2013;17(8):379-90 Available from: https:// www.ncbi.nlm.nih.gov/pmc/articles/PMC3624763/pdf/nihms412728.pdf.

67. Haier RJ, Karama S, Leyba L, Jung RE. MRI assessment of cortical thickness and functional activity changes in adolescent girls following three months of practice on a visual-spatial task. BMC Res Notes [Internet]. 2009;2(1):174. Available from: http://bmcresnotes.biomedcentral.com/ articles/10.1186/1756-0500-2-174.

68. Cardillo R, Vio C, Mammarella IC. A comparison of local-global visuospatial processing in autism spectrum disorder, nonverbal learning disability, ADHD and typical development. Res Dev Disabil. 2020;103(April):103682. Available from. https://doi.org/10.1016/j.ridd.2020.103682.

69. Bernardino I, Mouga S, Almeida J, Van Asselen M, Oliveira G, CasteloBranco M. A direct comparison of local-global integration in autism and other developmental disorders: implications for the central coherence hypothesis. PLoS One. 2012;7(6):e39351. Available from:. https://doi.org/ 10.1371/journal.pone.0039351.

70. Adolphs R. The social brain: neural basis of social knowledge. Annu Rev Psychol [Internet]. 2009;60(1):693-716 Available from: http://www.annua Ireviews.org/doi/10.1146/annurev.psych.60.110707.163514.

71. Schuck NW, Cai MB, Wilson RC, Niv Y. Human orbitofrontal cortex represents a cognitive map of state space. Neuron [Internet]. 2016;91(6):140212. Available from: https://linkinghub.elsevier.com/retrieve/pii/S0896 627316305116

72. Kana RK, Keller TA, Minshew NJ, Just MA. Inhibitory control in high-functioning autism: decreased activation and underconnectivity in inhibition networks. Biol Psychiatry. 2007;62(3):198-206.

73. Graewe B, Lemos R, Ferreira C, Santana I, Farivar R, De Weerd P, et al. Impaired processing of 3D motion-defined faces in mild cognitive impairment and healthy aging: an fMRI study. Cereb Cortex [Internet]. 2013;23(10):2489-99 Available from: https://academic.oup.com/cercor/ article-lookup/doi/10.1093/cercor/bhs246.

\section{Publisher's Note}

Springer Nature remains neutral with regard to jurisdictional claims in published maps and institutional affiliations.
Ready to submit your research? Choose BMC and benefit from:

- fast, convenient online submission

- thorough peer review by experienced researchers in your field

- rapid publication on acceptance

- support for research data, including large and complex data types

- gold Open Access which fosters wider collaboration and increased citations

- maximum visibility for your research: over $100 \mathrm{M}$ website views per year

At $\mathrm{BMC}$, research is always in progress.

Learn more biomedcentral.com/submissions 